OPEN ACCESS

Edited by:

Mihajlo (Michael) Jakovljevic,

Hosei University, Japan

Reviewed by:

Suresh Munuswamy,

Public Health Foundation of India, India

Seyi Lagoke,

South Western Sydney Local Health

District, Australia

${ }^{*}$ Correspondence:

Ruey-Hsia Wang

wrhsia@kmu.edu.tw

Specialty section: This article was submitted to

Health Economics,

a section of the journal Frontiers in Public Health

Received: 11 May 2021 Accepted: 21 June 2021

Published: 15 July 2021

Citation:

Yien J-M, Wang $H-H$, Wang $R-H$, Chou F-H, Chen K-H and Tsai F-S

(2021) Effect of Mobile Health

Technology on Weight Control in

Adolescents and Preteens: A

Systematic Review and Meta-Analysis.

Front. Public Health 9:708321.

doi: 10.3389/fpubh.2021.708321

\section{Effect of Mobile Health Technology on Weight Control in Adolescents and Preteens: A Systematic Review and Meta-Analysis}

\author{
Jui-Mei Yien ${ }^{1,2}$, Hsiu-Hung Wang ${ }^{1}$, Ruey-Hsia Wang ${ }^{1,3 *}$, Fan-Hao Chou ${ }^{1}$, \\ Kuo-Hsiung Chen ${ }^{4}$ and Fu-Sheng Tsai ${ }^{4,5,6}$
}

${ }^{1}$ College of Nursing, Kaohsiung Medical University, Kaohsiung, Taiwan, ${ }^{2}$ Department of Health Care Management, University of Kang Ning, Tainan City, Taiwan, ${ }^{3}$ Department of Medical Research, Kaohsiung Medical University Hospital, Kaohsiung, Taiwan, ${ }^{4}$ Department of Business Administration, Cheng Shiu University, Kaohsiung, Taiwan, ${ }^{5}$ Center for Environmental Toxin and Emerging-Contaminant Research, Cheng Shiu University, Kaohsiung, Taiwan, ${ }^{6}$ Super Micro Mass Research and

Technology Center, Cheng Shiu University, Kaohsiung, Taiwan

Childhood obesity is a crucial public health concern. In recent years, numerous studies have employed mobile health technology applications for weight control in children but obtaining varying effects. We conducted a meta-analysis to discuss the effectiveness of mobile health technology in reducing the body mass index (BMI) of obese children. The standardized mean difference (SMD) in BMl between the intervention and control groups was employed to measure the effect of mobile health technology intervention on weight control. The Comprehensive Meta-Analysis Version 3 software was employed for meta-analysis, and the results are presented in a forest plot. This study included nine randomized control trials, which featured a total of 1,202 participants. The meta-analysis revealed that mobile health technology intervention did not have a significant weight loss effect on subjects with obesity. However, by using ethnicity as a moderating variable for subgroup analysis, we discovered that the BMI of ethnic Chinese groups who received mobile health technology intervention was significantly lower than that of the control group. This effect was not significant in the non-ethnic Chinese subgroup. Therefore, the use of mobile health technology intervention for weight control in ethnic Chinese children resulted in significantly lower BMI in these children; however, the use of mobile health technology intervention for weight control is unsuitable for non-ethnic Chinese children.

Keywords: childhood obesity, overweight, adiposity, digital device, adolescents, preteens

\section{INTRODUCTION}

Childhood obesity is a crucial, global health problem that has raised concerns among public health experts. However, the prevalence rate of childhood obesity in recent years has remained unchanged (1). Different from traditional weight loss intervention, which involves face-to-face health education, scholars have endeavored to identify alternate methods to provide enhanced weight loss effects $(2,3)$. Children and adolescents frequently use mobile health technologies to retrieve information from the Internet and interact with online communities in their daily lives; 
therefore, mobile health technologies are a feasible channel for providing health information (4). Recently, mobile health technologies have been applied to manage children's weight and prevent obesity (5). Some scholars have done similar studies before, but the included literature includes non-randomized trials (6). Randomized controlled trials (RCTs) are the inclusion criteria for this study. The database search date is also the most recent; therefore, there is newer discovery in our research.

Due to limited time and resources, health experts cannot timely monitor and support patients in their everyday lives. This limitation can be overcome by the development of self-health management technologies (6). Because technology is commonly used in the daily lives of most people, the use of mobile health technologies for weight control presents advantages of high accessibility and adherence (7). In theory, the use of mobile health technologies for weight control in children is effective; after intervention, real-time feedback can be obtained, which maintains the motivation for weight loss in users (8). Mobile health technology is an acceptable and feasible method for reducing childhood obesity. However, large heterogeneity in research design were found across different studies $(9,10)$. Therefore, a consensus regarding the efficiency of mobile health technology applications in weight control in children has yet to be reached (11). This may or may not be true. However, it is true that despite the contentions there are many studies demonstrating the effectiveness of these technologies. In fact, some groups have cautiously supported their use as an adjunct to weight management interventions. Therefore, the objective of this study was to examine the effect of mobile health technology on weight control in adolescents and preteens.

\section{METHODS}

\section{Search Strategy and Inclusion Criteria}

A meta-analysis was conducted to evaluate the weight control effect of mobile health technology on subjects with obesity. The researchers searched the Embase, Medline, Cochrane Library, Web of Science, and ScienceDirect databases for articles on mobile health technology interventions among subjects with obesity. The literature collection period ended in February, 2021; the search methods involved using suitable controlled vocabulary and free-text terms. The keywords used for searching included childhood obesity, pediatric obesity, electronic technology, smartphone, activity tracker, mobile device, mobile application, and fitness tracker. The search syntaxes used are detailed in the Appendix. Inclusion criteria include RCTs or comparative experimental research. All searched studies comprised at least two participant groups, namely the intervention group and control group. The target population consisted of children and adolescents with obesity risks. In the included literature, intervention was conducted during the follow-up period by using smartphones, social medial, or follow-up instruments.

The selected studies (including RCTs and review articles) were manually screened to determine their inclusion or exclusion in the final analysis. In the first phase, article titles and abstracts were reviewed, and single-arm studies, case series, and case reports were excluded. In the second phase, the researchers reviewed the entire articles, and exclusion criteria included the following: nonRCTs and studies with participants who were older than 18 years of age, absence of BMI data, studies containing participants with physical disability, and interventions not involving mobile health technology. In addition, childhood obesity caused by drug use or diseases was not included in the scope of this study.

\section{Data Extraction and Quality Assessment}

After the selected articles underwent a review by the two reviewers, the researchers used a predetermined format to extract article data, primarily the first authors, study periods, sample sizes, participant characteristics, and intervention contents. The two reviewers independently used the Jadad scale to evaluate the quality of the RCTs (12). Jadad scoring is generated across three dimensions, namely randomization (2 points), blinding ( 2 points), and withdrawals and dropouts (1 point). The total score ranges from 0 to 5 ; higher scores indicate higher literature quality $(12,13)$. If the reviewers' opinions differed, a discussion was held between the reviewers under the coordination of a corresponding author until a consensus was reached.

\section{Data Integration and Analysis}

$\mathrm{BMI}$ is the most commonly used obesity evaluation index and is used by the World Health Organization (WHO) (14), rendering it suitable for meta-analysis; it consists of simple calculations and easily accessible data (15). BMI mean and standard deviation are viable for statistical analysis. Therefore, we used BMI to evaluate weight loss effects. In particular, we used the postintervention standardized mean differences (SMDs) between the intervention and control groups to evaluate the intervention effect. The 95\% confidence interval (CI) of the overall SMD was negative, indicating that the weight loss effect in the intervention group was greater than that in the control group. The Comprehensive Meta-Analysis Version 3 (Biostat, Englewood, NJ, USA) was used for analysis. We used $I^{2}$ to assess the degree of heterogeneity among the studies. When $I^{2}>50 \%$, the included studies had high heterogeneity (16). The random-effect model was used to compile the SMDs in all selected studies and to further identity variables for subgroup analysis. In addition, Egger's test was used to determine potential publication bias. Statistical significance was set as $p<0.05$ (17); if $\mathrm{p}$ was not significant, the number of the included studies was sufficient for meta-analysis.

\section{RESULTS}

\section{Literature Search and Characteristics of Included Studies}

The article selection was conducted in accordance with the Preferred Reporting Items for Systematic Reviews and MetaAnalysis (PRISMA) (Figure 1). A total of 254 citations (97, 83, and 74 from Embase, Medline, and Cochrane Library, respectively) were collected. After exclusion of 55 repeated citations, 199 remained. After a review of the titles and abstracts, 74 unsuitable citations were eliminated. Finally, the researchers reviewed the full text of 125 studies. By applying the exclusion criteria, the researchers excluded 50 non-RCT studies, 20 studies with participants over 18 years old, 26 studies that did not present 


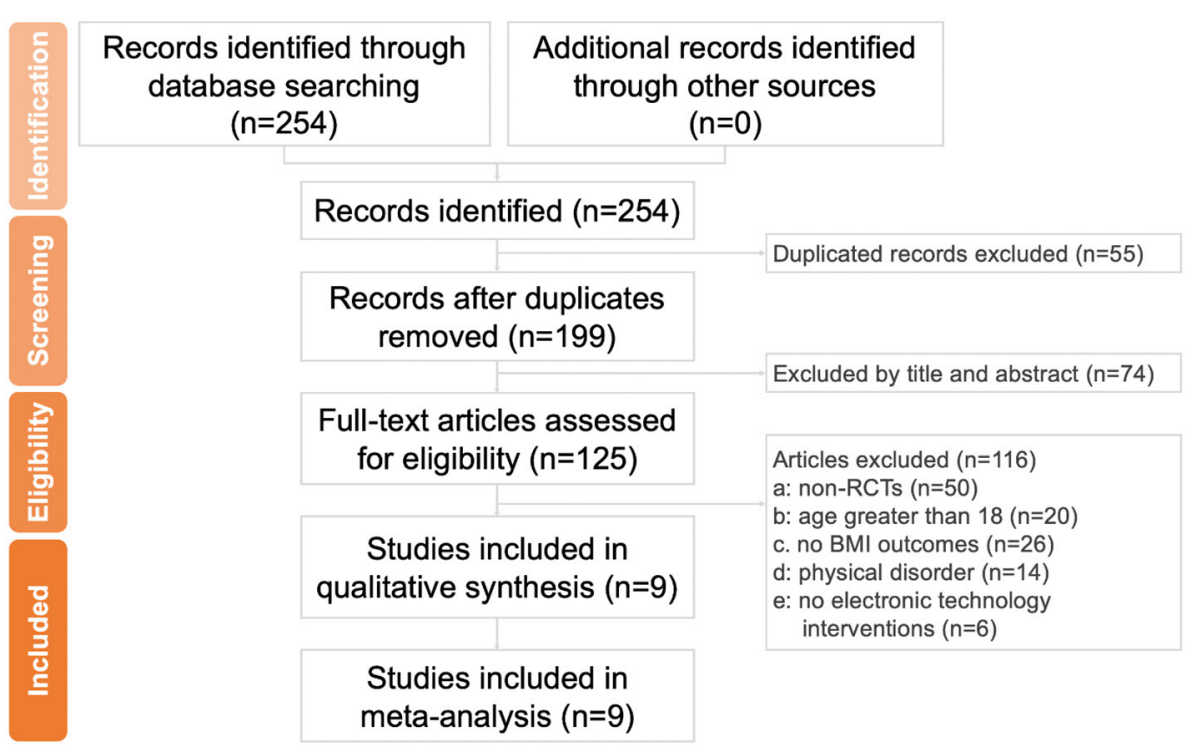

FIGURE 1 | Flow diagram of the study selection process.

their results in terms of BMI, 14 studies in which participants with physical disorders were involved, and 6 studies that did not use mobile health technology inventions. Finally, only 9 doublearm RCT studies were retained for meta-analysis. Regarding participant ethnicity, 3 studies conducted experiments on ethnic Chinese participants from the United States or Hong Kong (1820) based on sample descriptions of studies; 6 studies were based on non-ethnic Chinese participants from the United States $(21,22)$, Australia (23-25), and Italy (26).

Finally, 1,202 participants from the included 9 studies were included in the quantified analysis. Overall, the participant age ranged from 8 to 18 years; it ranged from 8 to 18 years for ethnic Chinese participants and 9 to 17 years for non-ethnic Chinese participants. The mobile health technology most often used by the participants included smartphones, wristbands, social media, and short message services, through which participants could engage in weight loss activities and report data. Table 1 lists the characteristics of the participants, the research method used in each study, and the Jadad scores of the included studies. The table reveals that the Jadad scores of the included studies were all $>4$, indicating favorable research quality.

\section{SMDs for Weight Control}

The meta-analysis included nine studies on weight loss in children. Figure 2 indicates that the range of $95 \%$ CI is shorter and the weighted values are greater for studies with larger sample size. In Figure 2, the overall SMD is -0.213 (95\% CI: -0.458 to 0.032 ). The lower and upper limits of the $95 \% \mathrm{CI}$ of the overall SMD crossed the vertical center line $(\mathrm{SMD}=0.00)$, indicating that the differences between the intervention groups and their corresponding control groups were non-significant. Subsequently, we computed the overall heterogeneity of the 9 included studies; 25,50 , and $75 \%$ heterogeneity represented low, moderate, and high heterogeneity, respectively (27). Because $I^{2}$ $=72.56 \%$, the studies exhibited high heterogeneity; therefore, identifying moderating variables for subgroup analysis is necessary. After carefully reviewing these nine studies, there seemed to be a discrepancy in effect on BMI between ethnic Chinese and non-ethnic Chinese subgroups. Therefore, we computed the heterogeneity levels between ethnic Chinese and non-ethnic Chinese participant groups and obtained results of $I^{2}<0.01 \%$ and $I^{2}=1.73 \%$, respectively. This indicates that both subgroups exhibited low heterogeneity, thereby verifying that ethnicity is a suitable variable for subgroup analysis. The analysis results in Figure 3 show that the 95\% CI of the overall SMD in the ethnic Chinese subgroup was situated to the left of the center line and did not cross it. This indicates that in the ethnic Chinese subgroup, the decrease in BMI in the intervention groups was significantly higher than that in the control groups (SMD: -0.773 ; 95\% CI: -1.069 to -0.476 ). In addition, Figure 4 indicates that the weight loss effect was not significantly different between the intervention and control groups of the non-ethnic Chinese participants (SMD: 0.019; 95\% CI: -0.106 to 0.145). Regarding the publication bias of the overall SMD, the results of Egger's test did not exhibit significance $(p=0.146)$, thereby indicating that publication bias was not present and that the number of included studies was sufficient for the meta-analysis.

\section{DISCUSSION}

This study conducted a meta-analysis to determine the effect of mobile health technology application intervention on weight loss in children. The meta-analysis included 3 and 6 studies on experiments conducted with ethnic Chinese and non-ethnic Chinese participants, respectively. The meta-analysis results indicated that mobile health 
TABLE 1 | Characteristics of the studies included.

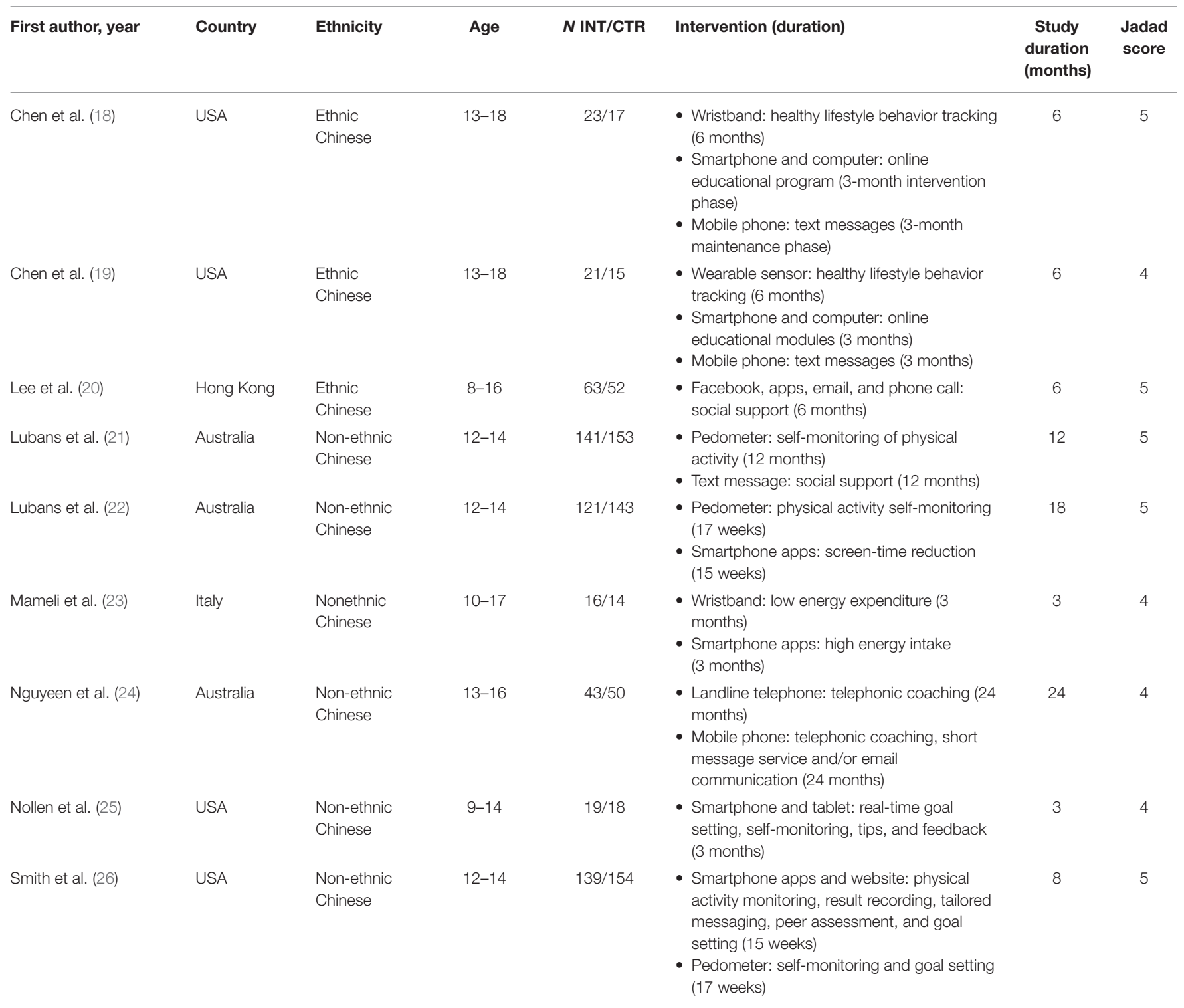

N, Number of participants; INT, Intervention; CTR, Control.

technology intervention did not have a significant effect on weight loss in children. However, when ethnicity was set as the variable for subgroup analysis, the BMI value in the ethnic Chinese intervention group was lower than that in the ethnic Chinese control group, thereby indicating a significantly greater weight loss effect in the ethnic Chinese intervention group. However, for non-ethnic Chinese participants, the intervention effect was not significant. This indicates that the weight loss intervention may consist of underlying mechanisms unrelated to mobile health technology intervention. We speculate that the intervention effects differed between ethnic Chinese subgroup and non-ethnic Chinese subgroup may be due to cultural factors, psychological factors, and the studies on ethnic Chinese participants having shorter follow-up periods than that of studies on non-Chinese participants.

From a cultural factor perspective, a possible mechanism that influences the intervention effect among ethnicity cultures is differences in body image and aesthetic perception (28). Differences in ethnicity culture result in different perceptions toward different body shapes. This factor may result in different obesity prevalence rates among different ethnicities (29). Ethnic Asian students in the United States exhibited high dissatisfaction toward their body image (30). Ethnic Chinese and non-ethnic Chinese adolescents had different psychological feelings toward body weight. Ethnic Chinese adolescents tended to be dissatisfied with their body weights and feel depressed; this phenomenon was not present in Hispanic adolescents (31). This may be the 


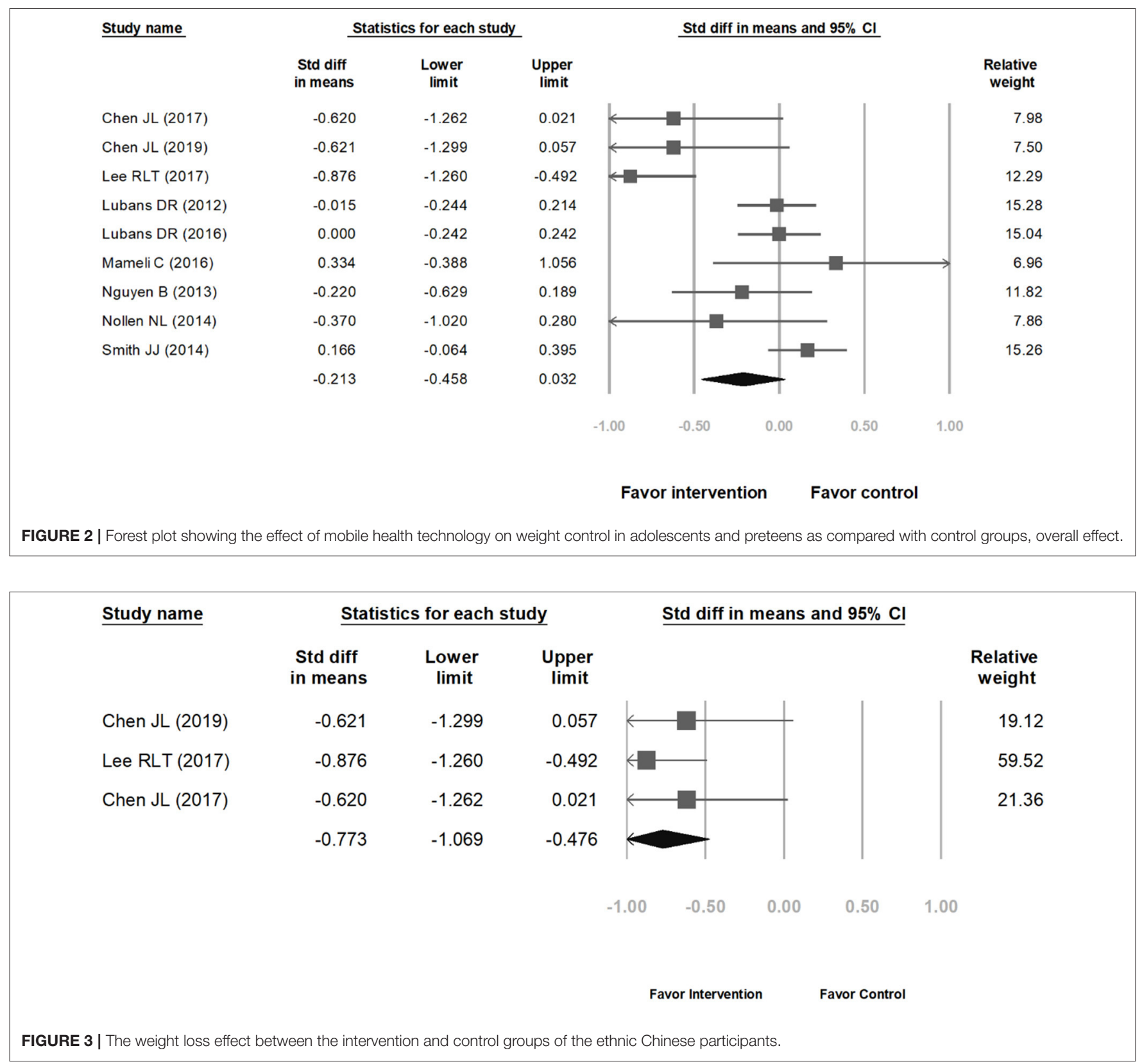

reason underlying the greater weight loss effect of mobile health technology intervention on ethnic Chinese children.

Unhealthy lifestyle is the main cause of obesity. The loss of motivation to live a healthy lifestyle results in an unbalanced diet and sedentary behavior among individuals (32). Maintaining a healthy body weight requires continuous effort; mobile health technology intervention is suitable for enhancing adolescents' motivation to maintain a regular diet and exercise habits (30). This indicates that in addition to the varying acceptance levels of different ethnicity groups toward mobile health technology intervention, differences in psychological factors among different ethnicities may result in varying weight loss effects $(33,34)$.
One of the important aspects of these technologies is not only that they are based on the evidence and that they support self-monitoring and goal setting, but that they also provide a sufficient amount or dose of "change talk"-that is motivational cognitive challenges that assist the adolescent in making changes in their behavioral choices and weight related decisions with the knowledge and experience that they inherently have and accrue through the intervention.

In addition, differences in the follow-up period in each study may have resulted in the significantly different temporal effects of weight loss (35). The follow-up period of the 3 studies conducted on ethnic Chinese participants was 6 months, whereas among the 6 studies conducted on non-ethnic Chinese participants, 4 


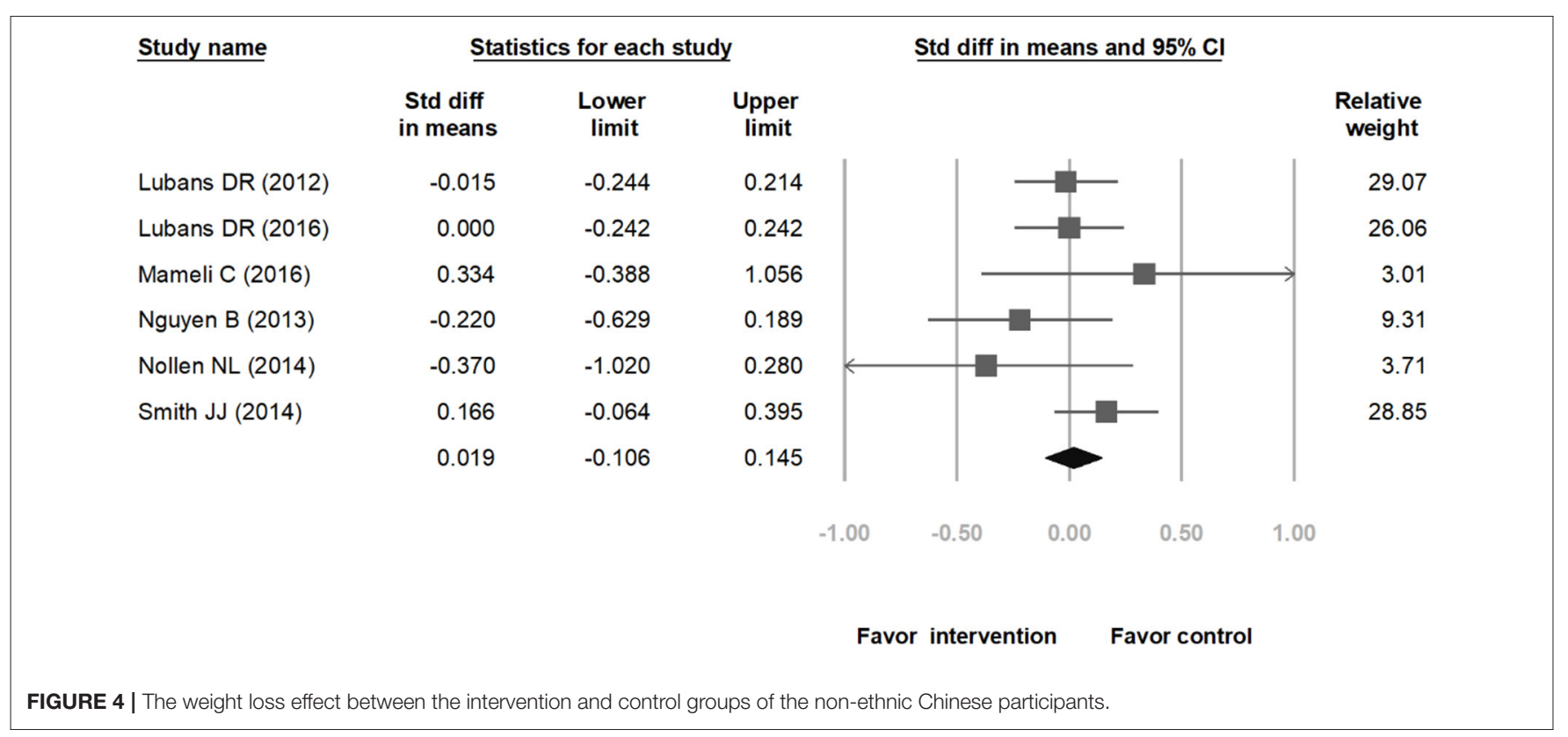

studies consisted of follow-up periods of $8,12,18$, and 24 months. Therefore, the weight loss effect of mobile health technology intervention on ethnic Chinese participants was only for a short term. Future studies on ethnic Chinese participants with longer follow-up periods are necessary. By using follow-up periods with the same duration, researchers can attain a greater understanding of the different effects of mobile health technology intervention on different ethnicity subgroups.

Based on the study results, we provide suggestions for future research. First, the meta-analysis of this study used mean BMI to measure weight loss results. Future studies may include other obesity measurement indices, such as BMI changes, zBMI, BMI\%, body fat, and waist-hip ratio. Next, the meta-analysis only included three studies involving ethnic Chinese participants. Future studies should include more studies on obesity in ethnic Chinese children. Finally, future researchers may conduct long-term follow-up on ethnic Chinese participants to fulfill the current research gap of longitudinal research.

\section{CONCLUSIONS}

Mobile health technology applications have been widely used in preventing childhood obesity. However, the results were not as expected. The research results indicate that by using ethnicity as a moderating variable and under the effect of cultural and psychological factors, the use of mobile health technology intervention may reduce obesity in ethnic Chinese children. However, the weight loss effect of mobile health technology intervention in other ethnicities remains uncertain. Therefore, ethnicity culture must be used as a key consideration in future studies using mobile health technology interventions to reduce childhood obesity.

\section{DATA AVAILABILITY STATEMENT}

The raw data supporting the conclusions of this article will be made available by the authors, without undue reservation.

\section{AUTHOR CONTRIBUTIONS}

R-HW, H-HW, and F-HC: conceptualization, methodology, and validation. J-MY: data curation, formal analysis, and writing original draft. J-MY and K-HC: investigation. R-HW: project administration, resources, and supervision. $\mathrm{H}-\mathrm{HW}$ and F-HC: resources. K-HC: software. F-ST: visualization, writing-review, and editing.

\section{SUPPLEMENTARY MATERIAL}

The Supplementary Material for this article can be found online at: https://www.frontiersin.org/articles/10.3389/fpubh. 2021.708321/full\#supplementary-material

\section{REFERENCES}

1. Nehus E, Mitsnefes M. Childhood obesity and the metabolic syndrome. Pediatr Clin. (2019) 66:31-43. doi: 10.1016/j.pcl.2018.0 8.004

2. Schroeder K, Travers J, Smaldone A. Are school nurses an overlooked resource in reducing childhood obesity? A systematic review and meta-analysis. J Sch Health. (2016) 86:309-21. doi: 10.1111/josh.12386

3. Bleich SN, Vercammen KA, Zatz LY, Frelier JM, Ebbeling CB, Peeters A. Interventions to prevent global childhood overweight 
and obesity: a systematic review. Lancet Diabetes Endo. (2018) 6:332-46. doi: 10.1016/S2213-8587(17)30358-3

4. Li JS, Barnett TA, Goodman E, Wasserman RC, Kemper AR. Approaches to the prevention and management of childhood obesity: the role of social networks and the use of social media and related electronic technologies: a scientific statement from the American Heart Association. Circulation. (2013) 127:260-7. doi: 10.1161/CIR.0b013e3182756d8e

5. Bailey LC, Milov DE, Kelleher K, Kahn, MG, Del Beccaro M, et al. Multiinstitutional sharing of electronic health record data to assess childhood obesity. PLoS ONE. (2013) 8:e66192. doi: 10.1371/journal.pone.0066192

6. Darling KE, Sato AF. Systematic review and meta-analysis examining the effectiveness of mobile health technologies in using selfmonitoring for pediatric weight management. Child Obes. (2017) 13:347-55. doi: $10.1089 /$ chi.2017.0038

7. Puig EP, Robles N, Saigí-Rubió F, Zamora A, Moharra M, Paluzie G, et al. Assessment of the efficacy, safety, and effectiveness of weight control and obesity management mobile health interventions: systematic review. JMIR mHealth uHealth. (2019) 7:12612. doi: 10.2196/12612

8. Lee J, Kim J. Development and efficacy testing of a social network-based competitive application for weight loss. Telemed J E Health. (2016) 22:4108. doi: $10.1089 / \mathrm{tmj} .2015 .0067$

9. Turner T, Spruijt-Metz D, Wen CF, Hingle MD. Prevention and treatment of pediatric obesity using mobile and wireless technologies: a systematic review. Pediatr Obes. (2015) 10:403-9. doi: 10.1111/ijpo.12002

10. Jia P, Xue H, Yin L, Stein A, Wang M, Wang Y. Spatial technologies in obesity research: current applications and future promise. Trends Endocrinol Metab. (2019) 30:211-23. doi: 10.1016/j.tem.2018.12.003

11. Triantafyllidis A, Polychronidou E, Alexiadis A, Rocha CL, Oliveira DN, da Silva AS, et al. Computerized decision support and machine learning applications for the prevention and treatment of childhood obesity: a systematic review of the literature. Artif Intell Med. (2020) 104:101844. doi: 10.1016/j.artmed.2020.101844

12. Jadad AR, Moore RA, Carroll D, Jenkinson C, Reynolds DJ, Gavaghan DJ, et al. Assessing the quality of reports of randomized clinical trials: is blinding necessary? Control Clin Trials. (1996) 17:1-12. doi: 10.1016/0197-2456(95)00134-4

13. Armstrong MJ, Mottershead TA, Ronksley PE, Sigal RJ, Campbell TS, Hemmelgarn BR. Motivational interviewing to improve weight loss in overweight and/or obese patients: a systematic review and meta-analysis of randomized controlled trials. Obes Rev. (2011) 12:09-723. doi: 10.1111/j.1467-789X.2011.00892.x

14. World Health Organization. BMI-for-Age (5-19 years). (2018). Available online at: https://www.who.int/toolkits/growth-reference-data-for-5to19years/indicators/bmi-for-age (accessed August 16, 2020).

15. Geserick M, Vogel M, Gausche R, Lipek T, Spielau U, Keller E, et al. Acceleration of BMI in early childhood and risk of sustained obesity. N Engl J Med. (2018) 379:1303-12. doi: 10.1056/NEJMoa 1803527

16. Kelishadi R, Haghdoost AA, Sadeghirad B, Khajehkazemi R. Trend in the prevalence of obesity and overweight among iranian children and adolescents: a systematic review and meta-analysis. Nutrition. (2014) 30:393400. doi: 10.1016/j.nut.2013.08.011

17. Yan J, Liu L, Zhu Y, Huang G, Wang PP. The association between breastfeeding and childhood obesity: a meta-analysis. BMC Public Health. (2014) 14:1267. doi: 10.1186/1471-2458-14-1267

18. Chen JL, Guedes CM, Cooper BA, Lung AE. Short-term efficacy of an innovative mobile phone technology-based intervention for weight management for overweight and obese adolescents: pilot study. Interact J Med Res. (2017) 6:e12. doi: 10.2196/ijmr.7860

19. Chen JL, Guedes CM, Lung AE. Smartphone-based healthy weight management intervention for chinese american adolescents: short-term efficacy and factors associated with decreased weight. J Adolesc Health. (2019) 64:443-9. doi: 10.1016/j.jadohealth.2018.08.022

20. Lee RLT, Leung C, Chen H, Louie LH, Brown M, Chen JL, et al. The impact of a school-based weight management program involving parents via mhealth for overweight and obese children and adolescents with intellectual disability: a randomized controlled trial. Int J Environ Res Public Health. (2017) 14:1178. doi: 10.3390/ijerph14101178
21. Lubans DR, Morgan PJ, Okely AD, Dewar D, Collins CE, Batterham M, et al. Preventing obesity among adolescent girls: one-year outcomes of the nutrition and enjoyable activity for teen girls (NEAT Girls) cluster randomized controlled trial. Arch Pediatr Adolesc Med. (2012) 166:8217. doi: 10.1001/archpediatrics.2012.41

22. Lubans DR, Smith JJ, Plotnikoff RC, Dally KA, Okely AD, Salmon J, et al. Assessing the sustained impact of a school-based obesity prevention program for adolescent boys: the ATLAS cluster randomized controlled trial. Int $J$ Behav Nutr Phys Act. (2016) 13:92. doi: 10.1186/s12966-016-0420-8

23. Mameli C, Brunetti D, Colombo V, Bedogni G, Schneider L, Penagini F, et al. Combined use of a wristband and a smartphone to reduce body weight in obese children: randomized controlled trial. Pediatr Obes. (2016) 13:8187. doi: 10.1111/ijpo.12201

24. Nguyen B, Shrewsbury VA, O'connor J, Steinbeck KS, Hill AJ, Shah $\mathrm{S}$, et al. Two-year outcomes of an adjunctive telephone coaching and electronic contact intervention for adolescent weight-loss maintenance: the loozit randomized controlled trial. Int J Obes. (2013) 37:46872. doi: $10.1038 /$ ijo. 2012.74

25. Nollen NL, Mayo MS, Carlson SE, Rapoff MA, Goggin KJ, Ellerbeck EF. Mobile technology for obesity prevention: a randomized pilot study in racial-and ethnic-minority girls. Am J Prev Med. (2014) 46:4048. doi: 10.1016/j.amepre.2013.12.011

26. Smith JJ, Morgan PJ, Plotnikoff RC, Dally KA, Salmon J, Okely AD, et al. Smart-phone obesity prevention trial for adolescent boys in lowincome communities: the ATLAS RCT. Pediatrics. (2014) 134:e723e31. doi: 10.1542/peds.2014-1012

27. Higgins JP, Thompson SG, Deeks JJ, Altman DG. Measuring inconsistency in meta-analyses. BMJ. (2003) 327:557-60. doi: 10.1136/bmj.327.7414.557

28. Kim KB, Aubrey JS. A cross-cultural comparison of cognitive and affective mediators in the relationship between media use and body image disturbance: focusing on US and Korean women. Asian J Commun. (2015) 25:50724. doi: $10.1080 / 01292986.2014 .995681$

29. Anderson PM, Butcher KF, Schanzenbach DW. Understanding recent trends in childhood obesity in the United States. Econ Hum Biol. (2019) 34:1625. doi: 10.1016/j.ehb.2019.02.002

30. Bucchianeri MM, Fernandes N, Loth K, Hannan PJ, Eisenberg ME, NeumarkSztainer D. Body dissatisfaction: do associations with disordered eating and psychological well-being differ across race/ethnicity in adolescent girls and boys? Cultur Divers Ethnic Minor Psychol. (2016) 22:13746. doi: $10.1037 / \mathrm{cdp} 0000036$

31. Xie B, Unger JB, Gallaher P, Johnson CA, Wu Q, Chou CP. Overweight, body image, and depression in Asian and hispanic adolescents. Am J Health Behav. (2010) 34:476-88. doi: 10.5993/AJHB.34.4.9

32. Evert AB, Franz MJ. Why weight loss maintenance is difficult. Diabetes Spectr. (2017) 30:153-6. doi: 10.2337/ds017-0025

33. Griffiths S, Angus D, Murray SB, Touyz S. Unique associations between young adult men's emotional functioning and their body dissatisfaction and disordered eating. Body Image. (2014) 11:175-8. doi: 10.1016/j.bodyim.2013.12.002

34. Bucchianeri MM, Serrano JL, Pastula A, Corning AF. Drive for muscularity is heightened in body-dissatisfied men who socially compare. Eat Disord. (2014) 22:221-32. doi: 10.1080/10640266.2013.874825

35. Stephens J, Allen J. Mobile phone interventions to increase physical activity and reduce weight: a systematic review. J Cardiovasc Nurs. (2013) 28:3209. doi: 10.1097/JCN.0b013e318250a3e7

Conflict of Interest: The authors declare that the research was conducted in the absence of any commercial or financial relationships that could be construed as a potential conflict of interest.

Copyright $\odot 2021$ Yien, Wang, Wang, Chou, Chen and Tsai. This is an open-access article distributed under the terms of the Creative Commons Attribution License (CC BY). The use, distribution or reproduction in other forums is permitted, provided the original author(s) and the copyright owner(s) are credited and that the original publication in this journal is cited, in accordance with accepted academic practice. No use, distribution or reproduction is permitted which does not comply with these terms. 


\section{APPENDIX}

\section{Embase}

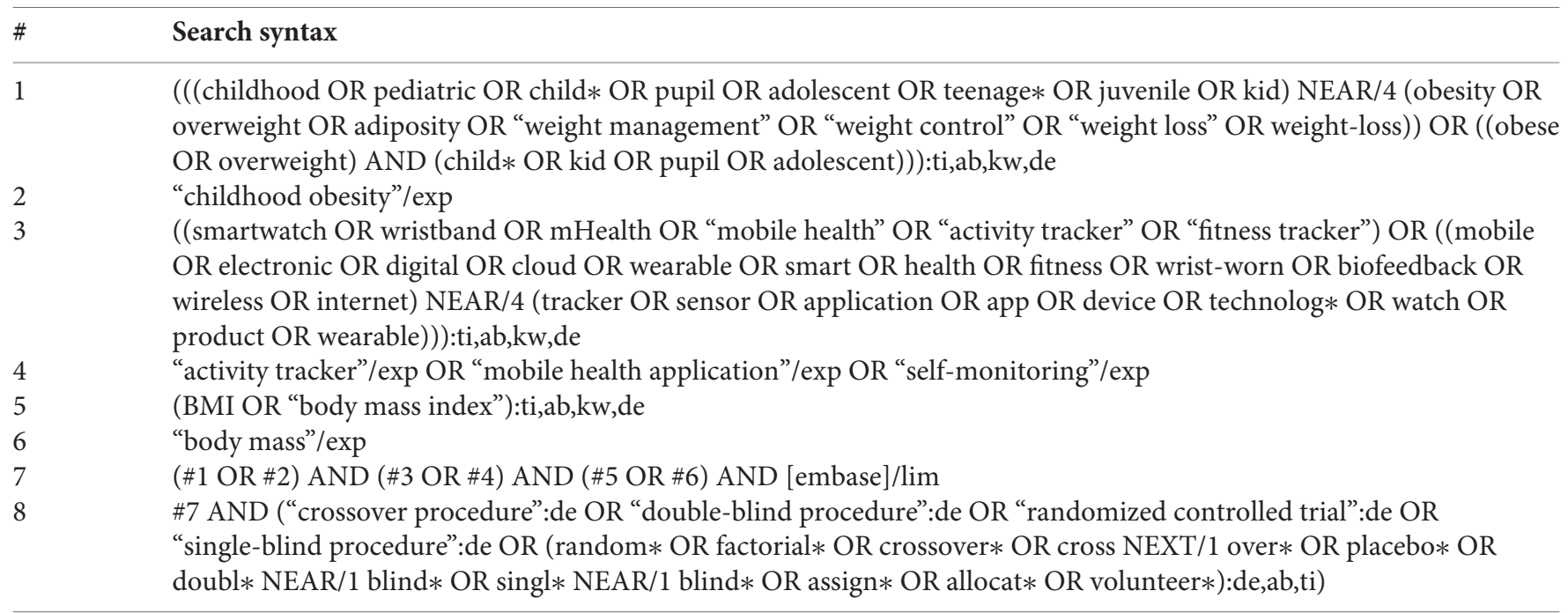

